\title{
Determinazione delle ipersuperficie che ammettono rappresentazioni geodetiche.
}

\author{
(Di Enruco Bomplant, a Roma.)
}

1. Una corrispondenza puntuale fra due varietà $V_{n}, V^{\prime}$ si dice geodetica (e le due varietà rappresentate geodeticamente una sull'altra) quando alle geodetiche di una di esse corrispondono quelle dell'altra.

Dato un elemento lineare generico esso non ammette rappresentazioni geodetiche che su elementi lineari uguali o che ne differiscono per una costante moltiplicativa; ciò̀ una $V_{n}$ generica non è geodeticamente rappresentabile che su quelle isometrico-simili ad essa.

Però come esistono tipi di superficie (di Liouvills) che ammeltono trasformazioni geodetiche, così esistono tipi di $V_{n}$ (tutti determinati dal prof. LeviCıvota) she ammettono trasformazioni geodetiche proprie (cioè non prodotti di isometrie per similitudini).

Nel caso delle $V_{n}(n>2)$ si presenta la questione (che ha sempre risposta affermativa per $n=2$ ) di vedere se esistano $V_{n}$ con trasformazioni geodetiche proprie in un $S_{n \vdash 1}$ euclideo, cioè ipersuperficie possedenti elementi lineari del LEVI-Givita.

Mi è parso che la risoluzione di questa questione riuscisse interessante per la conoscenza più approfondita delle $V_{n}$ con trasformazioni geodetiche; e perciò la presento qui per le $V_{3}$ di $S_{4}$ ritenendo non vi siano difficoltà molto maggiori per le $V_{n}$ di $S_{n+1}$.

La trattazione consiste nel ricercare quali condizioni impongono ai coefficienti degli elementi lineari di Levi-Givita le equazioni di Gauss e di CoDAZZ (quali si trovano nelle classiche Lezioni di Geometria differenziale del prof. L. BıаNchi, vol. I, alle quali il lettore è pregato di riferirsi senza bisogno di citazioni particolari).

Nella determinazione delle $V_{3}$ deformabili in $S_{4}$ (cioè per le quali la seconda forma fondamentale non è completamente determinata dalla prima) 
poteva supporsi che fosse necessario ricorrere alla Memoria del Braxch, Sulle varietò a tre dimensioni deformabili entro lo spazio euclideo a quattro dimensioni [Memorie della Soc. Ital. delle Scienze (detta dei XL), s. III, t. XIII, 1905]; ma dato il tipo particolare degli elementi lineari in esame ciò non è neppur necessario, perchè riesce senza difficoltà la costruzione di modelli sui quali la deformabilità è evidente.

Invece le $V_{3}$ rigide con trasformazioni geodetiche, esistenti in uno $S_{4}$ euclideo sono: le ipersfere, le iperquadriche (in particolare rotonde, intorno ad un piano principale, o a due piani principali sghembi fra loro), le ipersuperficie rotonde intorno ad una retta (e non intorno ad un piano, escluso il caso detto delle iperquadriche).

Ho poi completato la ricerca, del tutto analoga, aggiungendo la determinazione delle ipersuperficie con trasformazioni geodetiche proprie esistenti in spazi a curvatura costante, di cui ho pure assegnato i modelli.

1. Richiamı suleg equazioni di Gauss e di Codazz.

2. Com'è noto (Branchl, Lezioni cit., vol. I, pag. 362) un'ipersuperficie $V_{n-1}$ di uno spazio ad $n$ dimensioni è individuata rispetto al gruppo dei novimenti da due forme quadratiche

$$
\begin{aligned}
& d s^{2}=\sum_{i}^{n-1} a_{i k} d x_{i} d x_{k} \\
& \sum_{i}^{n} \sum_{i k}^{1} \Omega_{i k} d x_{i} d x_{k}
\end{aligned}
$$

i coefficienti delle quali sono legati dalle equazioni di Gauss e di Codazz che nel caso di un $S_{n}$ euclideo si scrivono

$$
\begin{gathered}
\Omega_{\alpha \beta} \Omega_{\gamma \delta}-\Omega_{\alpha \gamma} \Omega_{\beta \delta}=(\alpha \delta, \beta \gamma) \\
\frac{\partial \Omega_{\alpha \beta}}{\partial u_{\gamma}}-\frac{\partial \Omega_{\alpha \gamma}}{\partial u_{\beta}}+\sum_{1}^{n-1}|\alpha \beta| \Sigma_{t}\left|\Omega_{\gamma_{t}}-\sum_{1}^{n-1}\right| \alpha \gamma\left|{ }_{t}\right| \Omega_{\beta t}=0
\end{gathered}
$$

i simboli di Rremann e di Christoffec sono costruiti rispetto al $d s^{2}$ scritto. Nel caso di una $V_{3}$ di $S_{4}$ (posto $u_{1}=u, u_{2}=v, u_{3}=w$ ) e supposto, come 
accadrà nel nostro caso, il $d s^{2}$ del tipo

$$
d s^{2}=H_{1} d u^{2}+H_{2} d v^{2}+H_{3} d w^{2}
$$

si lta

$$
\begin{aligned}
(12,12)= & \left.1\left(\frac{\partial^{2} H_{1}}{\partial v^{2}}+\frac{\partial^{2}}{\partial} \frac{H_{2}}{u^{2}}\right)+\frac{1}{4} \frac{1}{H_{1}}\right)\left(\frac{\partial H_{2}}{\partial v}\right)^{2}+\frac{\partial H_{1}}{\partial u} \frac{\partial H_{2}}{\partial u}+ \\
& +\frac{1}{4} \frac{1}{H_{2}}\left(\frac{\partial H_{2}}{\partial u}\right)^{2}+\frac{\partial H_{1}}{\partial v} \frac{\partial H_{2}}{\partial v} \mid-\frac{1}{4} \frac{1}{H_{3}} \frac{\partial H_{1}}{\partial w} \frac{\partial H_{2}}{\partial w} \\
(12,23)= & \frac{1}{2} \frac{\partial^{2} H_{2}}{\partial u \partial w}-\frac{1}{4} \frac{1}{H_{1}} \frac{\partial H_{1}}{\partial w} \frac{\partial H_{2}}{\partial u}-\frac{1}{4} \frac{1}{H_{2}} \frac{\partial H_{2}}{\partial u} \frac{\partial H_{2}}{\partial w}-\frac{1}{4} \frac{1}{\partial H_{3}} \frac{\partial H_{3}}{\partial u} \frac{\partial H_{2}}{\partial w}
\end{aligned}
$$

quindi le equazioni di Gauss si scrivono:

$$
\begin{aligned}
& \left.\left.\Omega_{11} \Omega_{22}-\Omega_{12}^{2}=-\frac{1}{2} \mid \frac{\partial^{2} H_{1}}{\partial v^{2}}+\frac{\partial^{2} H_{2}}{\partial u^{2}}\right\}+\frac{1}{4} \frac{1}{H_{1}} \mid\left(\frac{\partial H_{1}}{\partial v}\right)^{2}+\frac{\partial H_{1}}{\partial u} \frac{\partial H_{2}}{\partial u}\right\}+ \\
& +\frac{1}{4} \frac{1}{H_{2}}\left|\left(\frac{\partial H_{2}}{\partial u}\right)^{2}+\frac{\partial H_{1}}{\partial v} \frac{\partial H_{2}}{\partial v}\right|-\frac{1}{4} \frac{1}{H_{3}} \frac{\partial H_{1}}{\partial w} \frac{\partial H_{2}}{\partial w} \\
& \Omega_{x 2} \Omega_{33}-\Omega_{23}^{2}=-\frac{1}{2}\left|\frac{\partial^{2} H_{2}}{\partial w^{2}}+\frac{\partial^{2} H_{3}}{\partial v^{2}}\right|+\frac{1}{4} \frac{1}{H_{2}}\left\{\left(\frac{\partial H_{2}}{\partial w}\right)^{2}+\frac{\partial H_{2}}{\partial v} \frac{\partial H_{3}}{\partial v}\right\}+ \\
& +\frac{1}{4} \frac{1}{H_{3}}\left\{\left(\frac{\partial H_{3}}{\partial v}\right)^{2}+\frac{\partial H_{2}}{\partial w} \frac{\partial H_{3}}{\partial w}\right\}-\frac{1}{4} \frac{1}{H_{1}} \frac{\partial H_{2}}{\partial u} \frac{\partial H_{3}}{\partial u} \\
& \left.\left.\Omega_{33} \Omega_{11}-\Omega_{13}^{2}=-\frac{1}{2} \mid \frac{\partial^{2} H_{3}}{\partial u^{2}}+\frac{\partial^{2} H_{1}}{\partial w^{2}}\right\}+\frac{1}{4} \frac{1}{H_{3}} \mid\left(\frac{\partial I_{3}}{\partial u}\right)^{2}+\frac{\partial H_{3}}{\partial w} \frac{\partial H_{1}}{\partial w}\right\}+ \\
& +\frac{1}{4} \frac{1}{H_{1}}\left|\left(\frac{\partial H_{1}}{\partial w}\right)^{2}+\frac{\partial H_{s}}{\partial u} \frac{\partial H_{1}}{\partial u}\right|-\frac{1}{4} \frac{1}{H_{2}} \frac{\partial H_{3}}{\partial v} \frac{\partial H_{1}}{\partial v} \\
& \Omega_{12} \Omega_{23}-\Omega_{13} \Omega_{22}=\frac{1}{2} \frac{\partial^{2} H_{2}}{\partial u \partial w}-\frac{1}{4} \frac{1}{H_{1}} \frac{\partial H_{4}}{\partial w} \frac{\partial H_{2}}{\partial u}-\frac{1}{4} \frac{1}{H_{2}} \frac{\partial H_{2}}{\partial u} \frac{\partial H_{2}}{\partial w}- \\
& -\frac{1}{4} \frac{1}{H_{3}} \frac{\partial H_{3}}{\partial u} \frac{\partial H_{2}}{\partial v} \\
& \Omega_{23} \Omega_{31}-\Omega_{21} \Omega_{35}=\frac{1}{2} \frac{\partial^{2} H_{3}}{\partial v \partial u}-\frac{1}{4} \frac{1}{H_{2}} \frac{\partial H_{2}}{\partial u} \frac{\partial H_{3}}{\partial v}-\frac{1}{4} \frac{1}{H_{3}} \frac{\partial H_{3}}{\partial v} \frac{\partial H_{3}}{\partial u}- \\
& -\frac{1}{4} \frac{1}{H_{1}} \frac{\partial H_{1}}{\partial v} \frac{\partial H_{3}}{\partial u} \\
& \Omega_{s_{1}} \Omega_{12}-\Omega_{32} \Omega_{11}=\frac{1}{2} \frac{\partial^{2} H_{1}}{\partial w \partial v}-\frac{1}{4} \frac{1}{H_{3}} \frac{\partial H_{3}}{\partial v} \frac{\partial H_{1}}{\partial w}-\frac{1}{4} \frac{1}{H_{1}} \frac{\partial H_{1}}{\partial w} \frac{\partial H_{1}}{\partial v}- \\
& -\frac{1}{4} \frac{1}{H_{2}} \frac{\partial H_{2}}{\partial w} \frac{\partial H_{1}}{\partial v}
\end{aligned}
$$


e quelle di Codazzi :

$$
\begin{aligned}
& \frac{\partial \Omega_{11}}{\partial v}-\frac{\partial \Omega_{12}}{\partial u}=\frac{\partial \log \sqrt{H_{1}}}{\partial v}\left(\Omega_{11}+\frac{H_{1}}{H_{2}} \Omega_{22}\right)+\frac{\partial \log \sqrt{H_{2} \mid H_{i}}}{\partial u} \Omega_{12}+ \\
& +-\frac{H_{1}}{H_{3}} \frac{\partial \log \sqrt{H_{1}}}{\partial w} \Omega_{23} \\
& \frac{\partial \Omega_{14}}{\partial w}-\frac{\partial \Omega_{13}}{\partial u}=\frac{\partial \log \sqrt{H_{1}}}{\partial w}\left(\Omega_{11}+\frac{H_{1}}{H_{3}} \Omega_{33}\right)+\frac{\partial \log \sqrt{H_{3} \mid H_{1}}}{\partial u} \Omega_{13}+ \\
& +\frac{H_{1}}{H_{2}} \frac{\partial \log \sqrt{H_{1}}}{\partial v} \Omega_{23} \\
& \frac{\partial \Omega_{22}}{\partial w}-\frac{\partial \Omega_{23}}{\partial v}=\frac{\partial \log \sqrt{H_{2}}}{\partial w}\left(\Omega_{22}+\frac{H_{2}}{H_{3}} \Omega_{33}\right)+\frac{\partial \log \sqrt{H_{3} ! H_{1}}}{\partial v} \Omega_{23}+ \\
& +\frac{H_{2}}{H_{1}} \frac{\partial \log \sqrt{H_{2}}}{\partial u} \Omega_{31} \\
& \frac{\partial \Omega_{22}}{\partial u}-\frac{\partial \Omega_{21}}{\partial v}=\frac{\partial \log \sqrt{H_{2}}}{\partial u}\left(\Omega_{22}+\frac{H_{2}}{H_{1}} \Omega_{11}\right)+\frac{\partial \log \sqrt{H_{1} H_{2}}}{\partial v} \Omega_{2,}+ \\
& +\frac{H_{2}}{H_{3}} \frac{\partial \log \sqrt{ } H_{2}}{\partial w} \Omega_{31} \\
& \frac{\partial \Omega_{33}}{\partial u}-\frac{\partial \Omega_{31}}{\partial w}=\frac{\partial \log \sqrt{H_{3}}}{\partial u}\left(\Omega_{33}+\frac{H_{3}}{H_{1}} \Omega_{11}\right)+\frac{\partial \log \sqrt{H_{1} \mid H_{3}}}{\partial w} \Omega_{31} \\
& \text {. } H_{H_{2}} \frac{\partial \log \sqrt{H_{3}}}{\partial v} \Omega_{12} \\
& \frac{\partial \Omega_{33}}{\partial v}-\frac{\partial \Omega_{32}}{\partial w}=\frac{\partial \log \sqrt{H_{3}}}{\partial v}\left(\Omega_{33}+\frac{H_{3}}{H_{2}} \Omega_{22}\right)+\frac{\partial \log \sqrt{H_{2} \mid H_{3}}}{\partial w} \Omega_{33}- \\
& +\frac{H_{8}}{H_{1}} \frac{\partial \log v / \overline{H_{3}}}{\partial u} \Omega_{12}
\end{aligned}
$$

E noto che date $H_{1}, H_{2}, H_{3}$ le equazioni $\left(G_{1}\right),\left(G_{2}\right)$ definiscono, in generale, le $\Omega_{i k}$ e allora la $V_{3}$ è indeformabile; affinchè poi esista effettivamente in $S_{4}$ occorre e basta che i valori così ricarati soddistino alle $\left(\mathrm{C}_{1}\right),\left(\mathrm{C}_{2}\right),\left(\mathrm{C}_{3}\right)$. Se invece le $\left(G_{1}\right),\left(G_{2}\right)$ non individuano le $\Omega_{i k}$, aliora la $V_{3}$ è deformabile sempre che siano soddisfatte in conseguenza le equazioni di Codazz.

3. Poichè nel seguito accalrà sempre che i secondi membri delle $\left(G_{2}\right)$ sono nulli, vediamo come si semplitichino in tal caso le equazioni precedenti. 
I). Se $(12,12) \cdot(23,23) \cdot(31,31) \quad \mid=0$ (in punti generici della $\left.V_{3}\right)$ è necessoriamente $\Omega_{11} . \Omega_{22} . \Omega_{33}=0 ; \Omega_{12}=\Omega_{33}=\Omega_{31}=0$.

In tal caso la $V_{3}$, se esiste in $S_{4}$, è indeformabile, a vendosi dalle $\left(G_{1}\right)$

$$
\begin{gathered}
\Omega_{11}^{2}=\frac{(12,12)(13,13)}{(23,23)} ; \Omega_{22}^{2}=\frac{(23,23)(21,21)}{(31,31)} \\
\Omega_{33}^{2}=\frac{(31,31)(32,332)}{(12.102)} .
\end{gathered}
$$

Le equazioni di Codazzi divengono

$$
\begin{aligned}
& \frac{\partial \Omega_{11}}{\partial v}=\frac{1}{H_{2}} \frac{\partial \log \sqrt{H_{1}}}{\partial v}\left(\Omega_{11} H_{3}+\Omega_{22} H_{1}\right) ; \frac{\partial \Omega_{11}}{\partial w}=\frac{1}{H_{3}} \frac{\partial \log \sqrt{H_{1}}}{\partial w}\left(\Omega_{11} H_{3}+\Omega_{33} H_{1}\right)\left(C_{11}\right) \\
& \frac{\partial \Omega_{22}}{\partial w}=\frac{1}{H_{3}} \frac{\partial \log \sqrt{H_{2}}}{\partial w}\left(\Omega_{22} H_{3}+\Omega_{33} H_{2}\right) ; \begin{array}{c}
\partial \Omega_{22} \\
\partial u
\end{array}=\frac{1}{H_{1}} \frac{\partial \log \sqrt{H_{2}}}{\partial u}\left(\Omega_{22} H_{1}+\Omega_{11} H_{2}\right)\left(\mathrm{C}_{2}\right) \\
& \partial \Omega_{33}=\frac{1}{H_{1}} \frac{\partial \log \sqrt{H_{3}}}{\partial u}\left(\Omega_{33} H_{1}+\Omega_{11} H_{3}\right) ; \quad \frac{\partial \Omega_{33}}{\partial v}=\frac{1}{H_{3}} \frac{\partial \log \sqrt{H_{3}}}{\partial v}\left(\Omega_{33} H_{2}+\Omega_{22} H_{3}\right) . \quad\left(\mathrm{C}_{3}\right) \\
& \partial u
\end{aligned}
$$

Se, e solo se, queste sono soddisfatte dai precedenti valori telle $\Omega_{i i}$ esiste in $S_{4}$ la $V_{3}$ di $d s^{2}=H_{1} d u^{2}+H_{2} d v^{2}+H_{3} d x^{3}$ ed è indeformabile.

II). Se uno dei simboli (12,12), (23,23), (31,31) è "ullo, ve ne sono almeno due nulli; se p. es. $(12,12)-1=0,(23,23)=(31,31)=0$, allora

$$
\Omega_{13}=\Omega_{23}=\Omega_{33}=0 \text { e } \frac{\partial H_{3}}{\partial u}=\frac{\partial H_{3}}{\partial v}=0
$$

Sia infatti $(31,31)=0$ e gli altri due $=0$. Moltiplicando la prima e terza (e poi la prima e la seconda) delle $\left(G_{2}\right)$, per essere $(12,12)-1=0$ e $(23,23)=1=0$ si ricava $\Omega_{13} \Omega_{23}=0, \Omega_{13} \Omega_{12}=0$, quindi o $\Omega_{13}=0$, orvero $\Omega_{13}=0$ e $\Omega_{23}=\Omega_{21}=0$. Nel primo caso, $\Omega_{13}=0$, dalle $\left(G_{2}\right), \Omega_{12} \Omega_{23}=\Omega_{21} \Omega_{33}=\Omega_{32} \Omega_{11}=0$ e dalla terza $\left(G_{1}\right) \Omega_{33} \Omega_{11}=0$ : ora se $\Omega_{11}=0$ avendosi dalla prima $\left(G_{1}\right) \Omega_{12}=1=0$ dovrebbe esserè $\Omega_{23}=\Omega_{33}=0$, ciò̀ $(23,23)=0$ contro l'ipotesi; mentre se $\Omega_{33}=0$ dalla seconda $\left(G_{1}\right) \Omega_{23}=1=0$, quindi $\Omega_{11}=\Omega_{12}=0$, cioè $(12,12)=0$ pure contro le ipotesi. Nel secondo caso $\Omega_{32}=\Omega_{12}=0, \Omega_{13}=1=0$, dalla prima $\left(\mathrm{G}_{2}\right) \Omega_{22}=0$, quindi $(12,12)=0,(23,23)=0$ contro l'ipotesi.

Se invece supponiamo due simboli nulli, p. es. $(23,23)=(31,31)=0$, ma $(12,12)=1=0$, dalla prima e terza di $\left(G_{2}\right)$ si ha $\Omega_{18} \Omega_{23}=0$; sia p. es. $\Omega_{13}=0$. 
Allora anche $\Omega_{33} \Omega_{11}=\Omega_{12} \Omega_{23}=\Omega_{21} \Omega_{33}=\Omega_{23} \Omega_{11}=0$, quindi o $\Omega_{23}=\Omega_{33}=0$, o $\Omega_{11}=\Omega_{12}=0$; ma dovendo essere $(12,12)+1=0$ l'unica soluzione possibile ѐ $\Omega_{13}=\Omega_{23}=\Omega_{33}=0$.

In queste condizioni le $\left(\mathrm{C}_{3}\right)$ dànno

$$
\begin{aligned}
& \frac{1}{H_{1}} \frac{\partial \log H_{3}}{\partial u} \Omega_{11}+\frac{1}{H_{2}} \frac{\partial \log H_{3}}{\partial v} \Omega_{12}=0 \\
& \frac{1}{H_{1}} \frac{\partial \log H_{3}}{\partial u} \Omega_{12}+\frac{1}{H_{2}} \frac{\partial \log H_{3}}{\partial v} \Omega_{22}=0
\end{aligned}
$$

e poichè $\Omega_{11} \Omega_{22}-\Omega_{12}^{2}=(12,12)=1=0$ necessariamente $\frac{\partial H_{3}}{\partial u}=\frac{\partial H_{3}}{\partial v}=0$.

Le àltre equazioni di Codazzı sono

$$
\left.\begin{array}{c}
\frac{\partial \Omega_{11}}{\partial v}-\frac{\partial \Omega_{12}}{\partial u}=\frac{\partial \log \sqrt{H_{1}}}{\partial v}\left(\Omega_{11}+\frac{H_{1}}{H_{2}} \Omega_{22}\right)+\frac{\partial \log \sqrt{H_{2} \mid H_{1}}}{\partial u} \Omega_{12} ; \\
\frac{\partial \log \Omega_{11}^{2}}{\partial w}=\frac{\partial \log H_{1}}{\partial w} \\
\frac{\partial \Omega_{22}}{\partial u}-\frac{\partial \Omega_{12}}{\partial v}=\frac{\partial \log \sqrt{H_{2}}}{\partial u}\left(\Omega_{22}+\frac{H_{2}}{\left.H_{1} \Omega_{11}\right)+\frac{\partial \log \sqrt{ } H_{1} \mid H_{2}}{\partial v} \Omega_{12}} ;\right. \\
\frac{\partial \log \Omega_{22}^{2}}{\partial v}=\frac{\partial \log H_{2}}{\partial w} .
\end{array}\right\}
$$

Essendo il determinante delle $\Omega_{i k}$ nullo, in questa categoria andranno ricercate le $V_{3}$ deformabili.

III). Se $(12,12)=(23,23)=(31,31)=0$, la $V_{s}$ è applicabile sopra uno $S_{3}$.

\section{§ 2. TIPI DI ELEMENTT LINEARI}

a tre variabill dotati di trasformazioni geodetiche phophie.

4. Dalla ricerca del prof. Levi-Givita ( $\left.{ }^{1}\right)$ risulta che si hanno due soli tipi di $d s^{2}$ appartenenti a $V_{s}$ con trasformazioni geodetiche proprie (non semplici prodotti di applicabilità per similitudini) $\left({ }^{2}\right)$. Il primo di essi (tipo

(1) Sulla trasformazione delle equazioni dinamiche [Ann. di Matem., 1896].

(2) Perchè sulle molteplicità delle radici dell'equazione cubica che interviene in quella teoria non possono farsi che tre ipotesi; e quella di una radice tripla corrisponde appunto ad isometrie e similitudini. 
di LiouviLle generalizzato) si può scrivere:

$$
\left.\begin{array}{rl}
d s^{2}=|U-V| \cdot|U-W| d u^{2} & +|V-U| \cdot|V-W| d v^{2}+ \\
& +|W-U| \cdot|W-V| d w^{2}
\end{array}\right\}
$$

ove $U, V, W$ sono funzioni rispettiv. della sola $u, v, v$. Esso è rappresentabile geodeticamente sull'altro

$$
\left.\begin{array}{rl}
d s^{2}=\left|\frac{1}{U}-\frac{1}{V}\right| \cdot\left|\frac{1}{U}-\frac{1}{W}\right| d u^{2} & +\left|\frac{1}{V}-\frac{1}{U}\right| \cdot\left|\frac{1}{V}-\frac{1}{W}\right| d v^{2}+ \\
& +\left|\frac{1}{W}-\frac{1}{U}\right| \cdot\left|\frac{1}{W}-\frac{1}{V}\right| d w^{2}
\end{array}\right\}
$$

Nella forma (1), e conseguentemente nella $\left(1^{\prime}\right)$, ci si può liberare dai segni di valore assoluto purchè sia assicurato (almeno per determinati campi di variabilità delle $u, v, w$ ) il carattere essenzialmente positivo dei suoi coefficienti.

A questa condizione non si può soddisfare prendendo $H_{1}=(U-V)(U-W)$, e per $H_{2}, H_{3}$ le espressioni dedotte dalla precedente con permutazioni circolari, perchè si avrebbe $H_{1} H_{2}+H_{2} H_{3}+H_{3} H_{1}=0$, il che è incompatibile con la condizione posta; si può invece prendere $H_{1}=(U-V)(U-W)$, $H_{2}=(V-W)(V-U)$ e $H_{3}=-(W-U)(W-V)\left(\right.$ e si ha $H_{1} H_{2}-H_{2} H_{3}-$ $\left.-H_{3} H_{1}=0\right)$ e risultano effettivamente $H_{1}, H_{2}, H_{3}$ positivi p. es. per $U>W>V($ o $U<W<V)$; mi servirò quindi di questa ultima determinazione delle $H_{i}$.

L'altro tipo di $d s^{2}$ è

$$
d s^{2}=(k-W) d \sigma^{2}+d w^{2}
$$

ove $d \sigma$ è un elemento binario (nelle sole $u, v), W(w)$ è tale che nel campo che si considera $W<k$ (costante); ridotto il $d \sigma^{2}$ a forma isoterma si ha

$$
d s^{2}=\mathcal{A}(u, v)\left(d u^{2}+d v^{2}\right)(k-W)+d w^{2}
$$

rappresentabile geodeticamente sull'altro

$$
d s^{\prime \prime}=\Lambda(u, v)\left(d u^{2}+d v^{2}\right) \frac{k-W}{h W}+\frac{d w^{2}}{W^{\prime \prime}}
$$

sicchè in ogni caso il $d s$ delle nostre $V_{3}$ è riducibile a forma ortogonale $\left(^{3}\right)$.

(3) Ciì non è più vero, in generale, per le $V_{n-1}$ di $S_{n}(n>4)$; quindi si potrebbe presentare in conseguenza qualche difficoltà nuova. 
\$ $3 . V_{3}$ esistenti in $S_{4}$ ecclideo (O A GURVatura costante) CON FLEMENTO LINEARE $d s^{2}=(U-V)(U-W) d u^{2}+(V-U)(V-W) d v^{2}-(W-U)(W-V) d w^{2}$.

5. Si ha.

$$
\begin{gathered}
H_{1}=(U-V)(U-W) ; \frac{\partial H_{1}}{\partial u}=U^{\prime}(2 U-V-W) ; \\
\frac{\partial H_{1}}{\partial v}=-V^{\prime}(U-W) ; \frac{\partial^{2} H_{t}}{\partial v^{2}}=-V^{\prime \prime}(U-W)
\end{gathered}
$$

e le analoghe; sicchè le espressioni dei simboli di Riemann sono:

$$
\begin{aligned}
& (12,12)=\frac{1}{2}(U-W) V^{\prime \prime}+\frac{1}{2}(V-W) U^{\prime \prime}-\frac{1}{4} \frac{1}{H_{1}}(V-W)(3 U-2 W-V) U^{\prime 2}- \\
& -\frac{1}{4} \frac{1}{H_{3}}(U-W)(3 V-2 W-U) V^{\prime 2}+\frac{1}{4} \frac{1}{H_{3}}(U-V)^{2} W^{\prime 2} \\
& (23,23)=\frac{1}{2}(V-U) W^{\prime \prime}-\frac{1}{2}(W-U) V^{\prime \prime}+\frac{1}{4} \frac{1}{\bar{H}_{2}}(W-U)(3 V-2 U-W) V^{\prime 2}+ \\
& +\frac{1}{4} \frac{1}{H_{3}}(V-U)(3 W-2 U-V) W^{\prime 2}-\frac{1}{4} \frac{1}{H_{1}}(V-W)^{2} U^{\prime 2} \\
& (31,31)=-\frac{1}{2}(W-V) U^{\prime \prime}+\frac{1}{2}(U-V) W^{\prime \prime}+\frac{1}{4} \frac{1}{H_{3}}(U-V)(3 W-2 V-U) W^{\prime 2}+ \\
& +\frac{1}{4} \frac{1}{H_{1}}(W-V)(3 U-2 V-W) U^{\prime 2}-\frac{1}{4} \frac{1}{H_{2}}(W-U)^{2} V^{\prime 2} \\
& (12,23)=(23,31)=(31,12)=0 .
\end{aligned}
$$

Le ultime tre relazioni indicano che le congruenze delle linee coordinate sono quelle definite in ogni punto dalle direzioni principali della $V_{3}$; sicchè, adottando una locuzione proposta dal Branchi $\left({ }^{4}\right)$ si può dire che le nostre $V_{\text {a }}$ sono spazi normali; e siccome questa stessa circostanza si presenta per .

(4) L. Branchr, Sugli spazi normali a tre dimensioni colle curvature principali costanti [Atti R. Accad. dei Lincei, Classe di Scienze, vol. XXV, 1916 1 . 
l'altro tipo di $d s^{2}$ che esamineremo in seguito e non dipende affatto dallo spazio d'immersione della $V_{3}$ rileviamo che:

Tutte le $V_{\mathrm{s}}$ che ammettono trasformate geodeliche proprie (cioe non soltanto isometrico-simili) sono spazi normali del Bianchi.

Una conseguenza del massimo interesse delle prime tre equazioni è la relazione

$$
(12,12)-(23,23)-(31,31)=0
$$

dalla quale si ricava che o tulti tre i simboli scritti sono differenti da zero, quindi anche le tre curvature principali; oppure se uno di essi è nullo, dovendo per il $1 .^{\circ} 3$, II) esserne nulli almeno due, lo sono tutti tre, e la $V_{\mathrm{s}}$ è applicabile sopra uno $S_{\sharp}$ euclideo. Escludiamo dal seguito questo caso evidente.

6. Sia dunque $(12,12)(23,23)(31,31)=\mid=0$.

Converrà porre per semplicità di scrittura

$$
(12,12)=A_{3}, \quad(23,23)=A_{1}, \quad(31,31)=A_{2} .
$$

Le equazioni di Codazzı, per le espressioni delle $\Omega_{i k}$ trovate al $\left.1 .^{0}: 3,1\right)$, si scrivono

$$
\left.\begin{array}{l}
\frac{\partial \log A_{2}}{\partial v}+\frac{\partial \log A_{3}}{\partial v}-\frac{\partial \log A_{1}}{\partial v}=\frac{1}{H_{2}} \frac{\partial H_{1}}{\partial v} \frac{A_{1}}{A_{2}}+\frac{1}{H_{1}} \frac{\partial H_{1}}{\partial v} \\
\frac{\partial \log A_{2}}{\partial w}+\frac{\partial \log A_{3}}{\partial w}-\frac{\partial \log A_{1}}{\partial w}=\frac{1}{H_{3}} \frac{\partial H_{1}}{\partial w} \frac{A_{1}}{A_{3}}+\frac{1}{H_{1}} \frac{\partial H_{1}}{\partial v}
\end{array}\right\}
$$

Si ottengono combinazioni immediatamente integrabili operando per sottrazione e per addizione sulla seconda equazione di ciascun gruppo e sulla prima del successivo. 
Nel primo modo, tenuto conto che $A_{1}+A_{2}=A_{3}$ si ha

$$
\begin{gathered}
\frac{\partial}{\partial w} \log \frac{A_{2}}{A_{1}}=\frac{W^{\prime}(U-V)}{(U-W)(V-W)} ; \quad \frac{\partial}{\partial u} \log \frac{A_{3}}{A_{3}}=\frac{U^{\prime}(V-W)}{(U-V)(U-W)} \\
\frac{\partial}{\partial v} \log \frac{A_{1}}{I_{3}}=\frac{V^{\prime}(W-U)}{(V-W)(V-U)} .
\end{gathered}
$$

Supponiamo $U^{\prime} V^{\prime} W_{-}^{\prime}=0$ (discuteremo poi l'ipotesi opposta). Integrando si ha

$$
\begin{gathered}
\frac{A_{2}}{A_{1}}=\varphi_{21}(u, v) \frac{U-W}{W-V} ; \quad \frac{A_{3}}{A_{2}}=\varphi_{32}(w, u) \frac{U-V}{U-W} \\
\frac{A_{1}}{A_{3}}=\varphi_{13}(u, w) \frac{V-W}{V-U}
\end{gathered}
$$

le funzioni per ora indeterminate $\varphi_{i k}(i=k)$ derono esser tali che $\varphi_{i k}=1, \varphi_{k i}$ e inoltre $\varphi_{21} \cdot \varphi_{32} \cdot \varphi_{13}=1$, sicchè si può porre

$$
\frac{A_{2}}{A_{1}}=\frac{y_{3}(v)}{y_{1}(u)} \frac{U-W}{W-V} ; \quad \frac{A_{3}}{A_{2}}=\frac{y_{3}(w)}{y_{2}(v)} \frac{U-V}{U-W} ; \quad \frac{A_{1}}{A_{3}}=\frac{y_{1}(u)}{y_{3}(w)} \frac{V-W}{V-U} .
$$

Se si sostituiscono le espressioni ora trovate nella relazione $A_{1}+A_{2}=A_{3}$ si ottengono le nuove relazioni

$$
\frac{y_{1}-y_{2}}{\tilde{U}-V}=\frac{y_{2}-y_{3}}{V-W}=\frac{y_{3}-y_{1}}{W-U}
$$

alle quali si può soddisfare in due modi :

$$
\begin{array}{lr}
1^{\circ} \text { caso: } & y_{1}=y_{3}=y_{3}=k \text { (costante) } \\
2 .^{\circ} \text { caso: } & y_{1} / U=y_{2} / V=y_{3} / W=k \text { (costante) }
\end{array}
$$

potendosi omettere nelle $y_{i}$ la stessa costante additiva, essendo le $U, V, W$ definite appunto a meno di una tale costante.

Combinando invęce per addizione le equazioni di Codazzr si ha

$$
\begin{gathered}
\frac{\partial}{\partial w} \log A_{3}=\frac{W^{\prime}}{H_{3}} \mid \frac{A_{2}}{A_{3}}(U-V)+V-W_{\mid} \\
\frac{\partial}{\partial u} \log A_{1}=\frac{U^{\prime}}{H_{1}}\left|\frac{A_{3}}{A_{1}}(W-V)+U-W\right| \\
\frac{\partial}{\partial v} \log A_{2}=\frac{V^{\prime}}{H_{2}}\left|\frac{A_{3}}{A_{2}}(W-U)+V-W\right|
\end{gathered}
$$

equazioni che bisogna integrare distingendo i due casi segnalati. 
7. Nel $1^{\prime \prime}$ caso $\left(y_{i}=k\right)$ le equazioni precedenti si riducono a

da cui

$$
\frac{\partial}{\partial w} \log A_{3}=\frac{W^{\prime}}{H_{3}^{-}}\left(U+V-\mathcal{U} W=\frac{\partial}{\partial w} \log H_{3} ; \ldots\right.
$$

$$
A_{3}=H_{12}(n, v) H_{3} \quad I_{1}=H_{23}(v, w) H_{1}, \quad A_{2}=H_{31}(w, n) H_{2}
$$

e poichè le $A_{i}$ sono $=-0$, anche $h_{i r}-1-0$.

Se si formano di nuovo i rapporti di due $A_{i}$ nell'ipotesi attuale si trova

$$
\left.\frac{h_{12}}{(U-V)^{2}}=\frac{h_{92}}{(V-W)^{2}}=\frac{h_{31}}{(W-U)^{2}}=h \text { (cost. }\right)
$$

e in conseguenza

$$
A_{3}=(12,12)=h H_{1} H_{3}, \quad A_{2}=(13,13)=h H_{1} H_{3}, \quad A_{1}=(23,23: 3)=h H_{2} H_{3} .
$$

Le $V_{3}$ attuali sono a curvatura costante $h$ (necessariamente positiva) quindi ipersfere dello $S_{4}$ : queste sono rappresentabili geodeticamente su $S_{3}$ (con proiezione dal centro). L'integrazione delle ultime equazioni, quando si sostituiscano ai simboli di Rimmann le loro espressioni date in principio del n. ${ }^{\circ} 5$, darebbe la forma di $U, V, W$, cioè del $d s^{2}$ del tipo di Lrouvilue relativo all'ipersfera.

8. Nel $2^{\circ}$ caso $\left(y_{1} / U=y_{2} / V=y_{3} / W=k\right)$ le ultime combinazioni delle equazioni di Codazzi divengono

e integrando

$$
\frac{\partial}{\partial w} \log A_{3}=\frac{W^{\prime}}{W H_{3}}\left(U V-W^{2}\right)
$$

$$
A_{3}=\frac{H_{3}}{W} \theta_{12}(u, v) ; \quad A_{1}=\frac{H_{1}}{U} \theta_{23}(v, w) ; \quad A_{2}=\frac{H_{2}}{V} \theta_{31}(v, v) .
$$

Per determinare le funzioni, per ora incognite, $\theta_{i k}$ si osserva che nel caso attuale è pure

quindi

$$
\frac{A_{2}}{A_{1}}=\frac{V}{U} \frac{U-W}{W-V} ; \quad \frac{A_{3}}{A_{2}}=\frac{W}{V} \frac{U-V}{U-W} ; \quad \frac{A_{1}}{A_{3}}=\frac{U}{W} \frac{V-W}{V-U}
$$

$$
\theta_{12}=h\left(\frac{U-V}{U V}\right)^{2} ; \quad \theta_{23}=h\left(\frac{V-W}{V W}\right)^{2} ; \quad \theta_{31}=h\left(\frac{U-W}{U W^{-}}\right)^{2}
$$


e finalmente

$$
\begin{gathered}
A_{3}=(12,12)=\frac{\| W H_{1} H_{2}}{(U V W)^{2}} ; \quad I_{1}=(y 3,23)=\frac{h U H_{2} H_{3}}{(U V W)^{2}} ; \\
A_{2}=(31,31)=\frac{h V H_{1} H_{3}}{(U V W)^{2}} .
\end{gathered}
$$

Notiamo le espressioni delle tre curvature principali

$$
k_{1}=\frac{h U}{(U V W)^{2}} ; \quad k_{2}=\frac{h V}{(U V W)^{2}} ; \quad k_{3}=\frac{h W}{(U V W)^{2}} .
$$

Per determinare effettivamente le $V_{3}$ in esame occorre sostituire le espressioni dei simboli di Rimmann (date al $n^{0} 5$ ) nelle ultime equazioni ed esaminare se il sistema così ottenuto è integrabile e infine ricavare da esso $U, I, W$.

Posto $u_{1}=u, u_{2}=v, u_{3}=v, U_{1}=U, U_{3}=V, U_{3}=W \mathrm{e}$

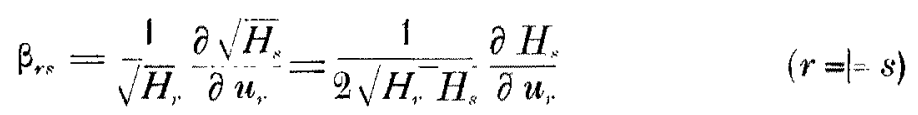

il sistema di equazioni cui debhono soddisfare le $\beta_{1: 8}$ è $\left({ }^{5}\right)$

$$
\begin{aligned}
& \frac{\partial \beta_{s s}}{\partial u_{t}}=\beta_{t+t} \beta_{t s} \\
& \frac{\partial \beta_{v s}}{\partial u_{v}}+\frac{\partial \beta_{s r}}{\partial u_{s}}+\beta_{t r} \beta_{t s}+k_{t} \sqrt{H_{,} H_{s}}=0 \\
& (r=|\cdot s-|=t)
\end{aligned}
$$

Le condizioni d'integrabilità nel caso attuale $\left(k_{t}\right.$ variabile) sono

e poichè

$$
\left(k_{t}-k_{s}\right) \frac{\partial \log H_{s}}{\partial u_{t}}+\left(k_{t}-k_{r}\right) \frac{\partial \log H_{v}}{\partial u_{t}}=-2 \frac{\partial k_{t}}{\partial u_{t}}
$$

$$
\begin{gathered}
k_{t} \cdots k_{s}=\frac{h}{\left(U_{*} U_{s} U_{t}\right)^{2}}\left(U_{t}-U_{s}\right) \\
\frac{\partial \log H_{s}}{\partial u_{t}}=\frac{U_{t}^{\prime}}{U_{t}-U_{s}} ; \quad \frac{\partial k_{t}}{\partial u_{t}}=-k_{t} \frac{\partial \log U_{t}}{\partial u_{t}}
\end{gathered}
$$

esse risultauo identicamente soddisfatte, quindi il sistema è completamente integrabile.

(5) L. Bianchr, Sugli spazi normali ecc., già citato. Le notazioni differiscono un poco perchè il BIANchr parte dal $d s^{2}=H_{1}^{2} d u_{1}^{2}+H_{2}^{2} d u_{2}^{2}+H_{3}^{2} d u_{3}^{2}$. 
Del resto riprendendo il sistema sotto la forma primitiva (senza l'intervento delle $\beta_{r s}$ )

$$
\begin{aligned}
& \frac{\hbar W H_{1} H_{2}}{(U V W)^{2}}=\frac{1}{2}(U-W) V^{\prime \prime}+\frac{1}{2}(V-W) U^{\prime \prime}- \\
& -\frac{1}{4} \frac{1}{H_{1}}(V-W)(3 U-2 W-V) U^{\prime 2}-\frac{1}{4} \frac{1}{H_{2}}(U-W)(3 V-2 W-U) V^{\prime 2}+ \\
& +\frac{1}{4} \frac{1}{4}(U-V)^{2} W^{\prime 2}
\end{aligned}
$$

$\frac{h U H_{2} H_{3}}{(\overline{U W})^{2}}=\frac{1}{2}(V-U) W^{\prime \prime}-\frac{1}{\mathbf{2}}\left(W^{r}-U\right) V^{\prime \prime}+$

$+\frac{1}{4} \frac{1}{H_{2}}(W-U)(3 V-2 U-W) V^{\prime 2}+\frac{1}{4} \frac{1}{H_{3}}(V-l)\left(3 V^{2}-U-V\right) W^{\prime 2}-$ $-\frac{1}{4} \frac{1}{H_{1}}(1-W)^{2} U^{\prime *}$

$\frac{h V H_{1} H_{s}}{(U V W)^{2}}=-\frac{1}{2}(W-V) U^{\prime \prime}+\frac{1}{2}(U-V) W^{\prime \prime}+\cdot$

$$
\begin{array}{r}
+\frac{1}{4} \frac{1}{H_{3}}(U-V)(3 W-2 V-U) W^{\prime \prime}+\frac{1}{4} \frac{1}{H_{1}}(W-V)(3 U-2 V-W) U^{\prime 2}- \\
-\frac{1}{4} \frac{1}{H_{2}}(W-U)^{2} V^{\prime 2}
\end{array}
$$

si constata senza difficoltà che le conseguenze differenziali

$$
\begin{aligned}
& \frac{1(U-V)^{2}}{2} W_{3}^{\prime \prime}-\frac{1}{2} U^{\prime \prime}-\frac{1}{2} V^{\prime \prime}=\frac{1}{4} V \frac{-U}{H_{1}^{2}}\left|2(U-W)^{2}+(U-V)^{2}\right| U^{\prime 2}+ \\
& +\frac{1}{4} U-V \mid 2(V-W)^{2}+(U-V)^{2} ! V^{\prime 2}+1 \frac{(U-V)^{2}}{4}(U+V-2 W) W^{\prime \prime}+ \\
& +\frac{h(U-V)^{2}}{(U \nabla W)^{2}}\left(U V-W^{2}\right) \\
& -\frac{1}{2} \cdot(W-V)^{2} U^{\prime \prime}+\frac{1}{H_{1}} \cdot V^{\prime \prime}-\frac{1}{2} W^{\prime \prime}=-\frac{1}{4} \frac{W-V}{H_{2}^{2}}, 9(r-U)^{2}+(W-V)^{2} \mid V^{\prime 2}- \\
& \left.-{ }_{4} \cdot \frac{W-V}{H_{3}^{2}}\right\} 2(W-U)^{2}+(W-V)^{2} \mid W^{\prime 2}-\frac{1}{4} \frac{(V-W)^{2}}{H_{1}^{2}}(2 U-r-W) U^{\prime 2}+ \\
& +\frac{h(W-V)^{2}}{(U V W)^{2}}\left(U^{2}-V W\right)
\end{aligned}
$$




$$
\begin{aligned}
& -\frac{1(U-W)^{2}}{2} V^{\prime \prime}-\frac{1}{2} W^{\prime \prime}+\frac{1}{2} U^{\prime \prime}=-\frac{1}{4}-H_{1}^{2}\left|2(U-V)^{2}+(W-U)^{2}\right| U^{2}- \\
& -\frac{1}{4} \frac{W-U}{H_{3}^{*}}\left|2(W-V)^{2}+(W-U)^{2}\right| W^{\prime 2}-\frac{1}{4} \frac{(W-U)^{2}}{H_{2}^{2}}(2 V-U-W) V^{\prime 2}+ \\
& +\frac{h(W-U)^{2}}{(U V W)^{2}}\left(V^{2}-U W\right)
\end{aligned}
$$

risultano conseguenze algebriche delle precedenti, quindi il sistema è integrabile.

La forma di queste ultime non è senza vantaggio per l'effettiva integrazione in quanto ciascuna di esse contiene due delle derivate seconde con coefficienti costanti; sicche mi servirò di queste piuttosto che delle equazioni di partenza.

Derivando p. es. la seconda rispetto ad $u$, l'espressione di $U^{\prime \prime \prime}$ contiene solo $V^{\prime z}$ e $W^{\prime 2}$ (delle derivate di $V$ e $W$ ) e così $U^{\prime \prime \prime}$ e $U^{r}$; talchè è da prevedere che si possano poi eliminare fra le tre equazioni così ottenute $V^{\prime 2}$ e $W^{\prime}$. Se si eseguiscono le operazioni indicate con qualche accorgimento spontaueo si trova

e analogamente

$$
\left[\frac{1}{U^{\prime}}\left(\begin{array}{l}
U^{\prime \prime \prime} \\
U^{\prime}
\end{array}\right)^{\prime}=-48 h U^{\prime} / U^{5}\right.
$$

$$
\left[\frac{1}{V^{\prime}}\left(\frac{V^{\prime \prime \prime}}{V^{\prime}}\right)\right]^{\prime}=-48 h V^{\prime} / V^{5},\left[\frac{1}{W^{\prime}}\left(\frac{W^{\prime \prime \prime}}{W^{\prime}}\right)^{\prime}\right]^{\prime}=48 h W^{\prime} / W^{5}
$$

(gli apici indicando derivazioni ordinarie); integrando si ha

$$
\begin{gathered}
U^{\prime 2}=-\frac{4 h}{D}+a_{1} U^{3}+a_{2} U^{2}+a_{3} U+a_{4} \\
V^{\prime 2}=-\frac{4 h}{V}+b_{1} V^{3}+b_{2} V^{2}+b_{3} V+b_{4} \\
W^{\prime 2}=+\frac{4 h}{W}+c_{1} W^{-3}+c_{2} W^{2}+c_{3} W+c_{4}
\end{gathered}
$$

con le $a_{i}, b_{i}, c_{i}$ costanti d'integrazione. Queste costanti non sono però tutte indipendenti come si rileva cercando di soddisfare con le espressioni ora trovate alle equazioni proposte.

Infatti, se si moltiplica la prima delle conseguenze algebrico-differenziali 
per $U-V$, tutti i termini di essa lo contengono a fattore meno i seguenti

$$
-\frac{1}{2} U^{\prime 2}+\frac{1}{2} V^{\prime 2}
$$

e poichè dunque occorre che anche quest'espressione sia divisibile per $U-V$ bisogna che sia $a_{i}=b_{i}$ : analogamente ragionando sopra una qualsiasi delle due rimanenti equazioni risulta ancora $a_{i}=b_{i}=-c_{i}$; sicchè posto

si ha

$$
P_{4}(t)=a_{1} t^{4}+a_{2} t^{3}+a_{3} t^{2}+a_{4} t-4 h
$$

$$
\int \frac{\sqrt{U}}{\sqrt{P_{4}(U)}} d U=u ; \quad \int \frac{\sqrt{V}}{\sqrt{P_{*}(V)}} d V=v ; \quad \int \frac{\sqrt{W}}{\sqrt{-P_{4}(W)}} d W=v
$$

potendosi scegliere sempre nulle le costanti che nascono dalle nuove integrazioni.

Non è detto (poichè non si è spinta a fondo la verifica delle equazioni differenziali) che le relazioni trovate fra le costanti d'integrazione siano le sole esistenti: ma ciò è di fatto, come risulta dal seguente ragionamento geometrico che ci risparmia non solo quella verifica, ma anche le integrazioni che sarebbero ancora necessarie per trovare la forma (cioè le equazioni parametriche o l'equazione cartesiana) delle $V_{3}$ in esame.

Poichè queste sono rigide basta trovare un modello con quell'elemento lineare per esser sicuri che la $V_{\mathrm{s}}$ esiste e coincide necessariamente col modello trovato: cioè che non ve ne sono altre.

Un modello è fornito dalle iperquadriche di $S_{4}$. Infatti il $d s^{2}$ di $S_{4}$ riferito al sistema 4-plo ortogonale di iperquadriche

è $\left({ }^{6}\right)$

$$
\frac{x^{2}}{\alpha-\tau}+\frac{y^{2}}{\beta-\tau}+\frac{z^{2}}{\gamma-\tau}+\frac{t^{2}}{\delta-\tau}=1
$$

$$
\begin{aligned}
& d s^{2}=-\frac{1}{4} \frac{(\lambda-\mu)(\lambda-\nu)\left(\lambda-n_{1}\right)}{(\alpha-\lambda)(\beta-\lambda)(\gamma-\lambda)(\delta-\lambda)} d \lambda^{2}- \\
& -1 \frac{(\mu-\lambda)(\mu-\nu)\left(\mu-\lambda_{1}\right)}{4-\mu)(\beta--\mu)(\gamma-\mu)(\delta-\mu)} d \mu^{*} \\
& -\frac{1}{4} \frac{(\nu-\lambda)(\nu-\mu)(\nu-n)}{(\alpha-\nu)(\beta-\nu)(\gamma-\nu)(\delta-\nu)} d \nu^{2}- \\
& -\frac{1}{4} \frac{(n-\lambda)(n-\mu)(n-\nu)}{(\alpha-n)(\beta-n)(\gamma-n)(\delta-n)} d n^{2}
\end{aligned}
$$

(6) Cfr. p. es. J. Sommth, Muth. Anmalen, Be. 53,1900, p. 113. 
con

$$
\alpha>n>\beta>v>\gamma>\mu>\delta>\lambda>-\infty
$$

quindi sull' iperquadrica $\mu=0$, p. es., si ha proprio un $d s^{2}$ del tipo voluto; e tanto basta per concludere che le nostre $V_{3}$ sono appunto iperquadriche. Anzi asseguato a piacere $P_{4}$ si hanno dal confronto del nostro elemento lineare con quest'ultimo i valori dei semiassi della relativa $V_{3}$ (risulta di qua nuovamente che se $h=0$ la $V_{3}$ è applicabile sopra un $S_{3}$ perchè tale è il modello). Se in particolare $a_{1}=0$ si hanno paraboloidi, come risulta dall'espressione del loro elemento lineare.

9. Prima di enunciare il risultato bisogna esaminare l'ipotesi finora scartata $U^{\prime} V^{\prime} W^{\prime}=0$.

Se uno solo dei tre fattori è nullo, facciamo $U^{\prime} V^{\prime}=0, W^{\prime}=0$. Poichè interessano le sole differenze fra $U, V, W$ si può fare $W=0$ (quindi $V<0$ ) e con un cangiamento di parametri $u, v$ possiamo partire dal

$$
d s^{2}=(U-V)\left(d u^{2}+d v^{2}\right)-U V d w^{2} .
$$

Per esso

$$
\begin{aligned}
& (12,12)=-\frac{1}{2}\left(U^{\prime \prime}-V^{\prime \prime}\right)+\frac{U^{\prime 2}+V^{\prime \prime}}{U-V^{\prime}} \\
& (23,23)=\frac{1}{2} U V^{\prime \prime}+\frac{1}{4 U-V} \frac{V}{U} U^{\prime 2}-\frac{1}{4(U-V) V} U V^{\prime \prime} \\
& (31,31)=\frac{1}{2} V U^{\prime \prime}-\frac{1-V V+2 U}{4(U-V) U} V U^{\prime 2}-\frac{1}{4} \frac{U}{U-V} V^{\prime 2}
\end{aligned}
$$

e gli altri simboli di Riemann sono $=0$. Posto $(12,12)=A_{3}, \ldots$, si ha

$$
\frac{A_{1}}{\bar{U}}-\frac{A_{2}}{\bar{V}^{\prime}}=A_{3}
$$

Delle equazioni di Codazz due sono identicamente soddisfatte; combimando le altre eome nel caso precedente si ha

$$
\frac{\partial}{\partial u} \log \frac{A_{3}}{A_{2}}=\frac{U^{\prime}}{U} \frac{V}{U-\gamma}: \frac{\partial}{\partial v} \log _{A_{1}}^{A_{3}}=-\frac{V^{\prime}}{V} \frac{U}{U-V}
$$

da cui

$$
\frac{A_{3}}{A_{\mathrm{q}}}=\frac{y_{2}(v)}{V} \frac{U-V}{U} ; \quad \frac{A_{3}}{A_{1}}=\frac{y_{1}(u)}{U} \frac{U-V}{V}
$$


sostituendo queste espressioni nella relazione fra le $A$, si ricara, designando con $k$ una costante,

$$
\text { (') } \quad \frac{A_{3}}{A_{1}}=\frac{A_{3}}{A_{2}}=\frac{Y-V}{U V} ;
$$

oppure

$$
\text { 2\%) } \quad \frac{A_{3}}{A_{2}}=\frac{k}{k-V} \frac{V \quad U}{U V} ; \quad \frac{A_{3}}{A_{1}}=\frac{k}{k-U} \frac{V-U}{U V} .
$$

Il primo caso conduce subito alle ipersfere; rimane da esaminare il secondo. Le equazioni rimaste di CoDazzr sono:

$$
\frac{\partial}{\partial u} \log A_{1}=\frac{U^{\prime}}{U-V}\left|1-V \frac{A_{3}}{A_{1}}\right| ; \quad \frac{\partial}{\partial v} \log A_{2}=-\frac{V^{\prime}}{U-V}\left|1+U \frac{A_{3}}{A_{2}}\right|
$$

e da queste integrando e servendosi delle relazioni già note fra le $A_{\text {; }}$

$$
A_{1}=\frac{h(k-U) H_{2} H_{3}}{(k-U)^{2}(k-V)^{2}} ; \quad A_{2}=\frac{h(k-V) H_{1} H_{3}}{(k-U)^{2}(k-V)^{2}} ; \quad A_{3}=\frac{h k H_{1} I_{2}}{(k-U)^{2}(k-V)^{2}}
$$

ove $h$ è una nuova coslante d'integrazione.

Notiamo ancora qui che, le $U$ e $V$ essendo definite a meno della stessa costante addittiva, si può da per tutto porre $U$ e $V$ in luogo di $U-k$ e $V-k$. Cosi facendo, e scritte al posto dei simboli $A_{i}$ le espressioni calcolate in principio di questo numero, si hanno tre equazioni differenziali (delle quali rue sole indipendenti); si verifica, come nel caso generale, che il sistema è completamente integrabile.

Dalla prima equazione, p. es.,

$$
\frac{h k(U-V)^{2}}{U^{2} V^{2}}=-\frac{1}{2}\left(U^{\prime \prime}-V^{\prime \prime}\right)+\frac{1 U^{\prime 2}+V^{\prime 2}}{2} U \frac{-\cdots V}{U}
$$

si ha con successive derivazioni rispetto ad $u$

$$
\left(U^{\prime \prime \prime} / U^{\prime}\right)^{\prime}=-12 h k U^{\prime} / U^{4}
$$

che integrata dà

$$
\int \frac{\sqrt{U} d U}{\sqrt{a_{1} U^{3}+a_{2} U^{2}+a_{3} U-4 h k}}=u
$$

(l'ultima costante d’integrazione si può fare $=0$ ). In modo analogo, e con 
osservazioni già esposte nel caso generale, si trova

$$
\int \frac{\sqrt{-V} d V}{\sqrt{a_{1} V^{3}}+\frac{a_{2} V^{3}+a_{3} V^{2}+4 h}{4 h}}=v .
$$

Le superticie $n=$ cost. nelle nostre $V_{3}$ hamo l'elemento lineare delle quadriche di $\varsigma_{3}$ soritho in coordinate ellittidhe; siceome $H_{1}, H_{2}, H_{3}$ non dipendono da $w$ si può pensare che le $r_{3}$ in esame siano quadriche rotonde intorno at un piano. E infalti presa una quadrica di $S_{z}$ riferita ai suoi piani principali come piani coordinati e detto $d a$ it suo elemento lineare, la $V_{3}$ che raste facendola ruotare in $14 n 0 \quad s_{4}^{t}$ intorno al piano $(y z)$ ha il

$$
d s^{2}=d \sigma^{2}+x^{2} d \theta^{2}
$$

( $d \theta$ misura la rotazione infinitesima); cioè in coordinate ellittiche (sulla quadrica di $S_{3}$ )

$$
\begin{aligned}
d s^{2}=\left.\right|_{4} ^{\prime}\left(\lambda_{1}-\lambda_{2}\right) ! \frac{\lambda_{1} d \lambda_{1}^{2}}{\mid\left(w^{2}+\right.} & \frac{\left.\lambda_{1}\right)\left(b^{2}+\lambda_{1}\right)\left(c^{2}+\lambda_{1}\right)}{\left(a^{2}+\lambda_{2}\right)\left(b^{2}+\lambda_{2}\right)\left(c^{2}+\lambda_{2}\right)} \mid+ \\
& +\frac{\left(a^{2}+\lambda_{1}\right)\left(a^{2}+\lambda_{2}\right) a^{2}}{\left(a^{2}-b^{2}\right)\left(a^{2}-c^{2}\right)} d 0^{2} ;
\end{aligned}
$$

il confronto fra il $d s^{2}$ di partenza e questo ci assicura (data la rigidità del modello del $d s^{2}$ ) che le nostre $V_{\mathrm{s}}$ sono quadriche rotonde intorno ad un piano (principale). Se negli integrali che dànoo $U, V$ è $a_{1}=0$, si hanno paraboloidi rotondi.

10. Veniamo all'ultimo caso rimasto da esaminare.

Se $U$ e $V$ sono costanti, mentre $W^{\prime}=0$, si può sempre, con eventuali cangiamenti di parametri, prender le mosse dal

$$
d s^{2}=(1+W) d u^{2}+(1-W) d v^{2}+d w^{2} .
$$

Per esso si ha

$$
\begin{aligned}
& (12,12)=\frac{1}{4} W^{\prime 2} \\
& (23,23)=\frac{1}{2} W^{\prime \prime}+\frac{1}{4} 1-W W^{\prime 2} \\
& (31,31)=\frac{1}{2} W^{\prime \prime}+\frac{1}{41+W} W^{\prime 2}
\end{aligned}
$$


da cui, con le solite notazioni, segue

$$
A_{1} A_{2}=\frac{2}{A^{2}}
$$

Le equazioni di Conazz non identicamente soddisfatte sono

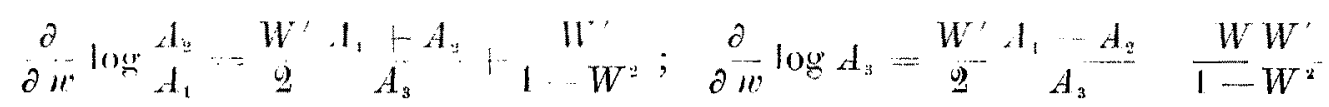

e dalla prima, tenuto conto della relazione fra le $A_{i}$, si ha integrando

$$
\frac{A_{2}}{A_{1}}=h \begin{aligned}
& 1+W \\
& 1-W
\end{aligned} \quad(h=\text { cost. }) .
$$

Se $h=1$, integrando la seconda equazione, si hanno le iporsfere; se invece $h==1$ posto $\frac{1+h}{1-h}=k$, la seconda equazione di Codazz si scrive

$$
\frac{\partial}{\partial w} \log A_{3}=\frac{\partial}{\partial w} \log (1+W)+(1-k) \frac{W^{\prime}}{(1-W)(k-W)}
$$

e da essa si trae

$$
A_{3}=c \frac{1-W^{2}}{k-\frac{W}{W}} \quad(c=\text { cost. }>0)
$$

e con le due relazioni già note si determinano poi $A_{1}$ e $A_{2}$. Resta da vedere se si possono soddisfare le equazioni di Rrmana con queste $A_{i}$.

Dalla prima si ha

$$
\frac{1}{4} W^{\prime q}=e \stackrel{1}{1-W}-W
$$

quindi

$$
\int \frac{\sqrt{k-W}}{\sqrt{1-W^{2}}} d W=2 \sqrt{c} w
$$

la costante dell'ultima integrazione potendosi prender nulla.

Le altre equazioni ne risultano conseguenza, sicchè il $d s^{2}$ è determinato. Questo elemento lineare appartiene, come facilmente si verifica, alle iperquadriche di $S_{4}$ rotonde intorno a due piani principali, ortogonali e incidenti in un sol punto (cioè alle iperquadriche di equazione $\frac{x^{2} i-y^{2}}{a^{2}} \pm \frac{z^{2}+t^{2}}{b^{2}}=1$ ); quindi queste sono le sole $V_{3}$ della nostra famiglia.

$\mathrm{E}$ con ciò la discussione è esaurita. 
11. Ne riassumiamo il risultato nel seguente teorema:

Le $V_{3}$ che commettono trasformazioni geodetiche proprie, con elemento lineare $d s^{2}=(U-V)(U-\cdots-W) d u^{2}+(V-U)(V \cdot W) d v^{2} \cdots(W \cdots U)(W-V) d w^{2}$ esistenti in uno $S_{4}$ enclideo (insieme a loro trusformate geodetiche) sono:

1) le ipersfere,

2) le iperquadriche in generale $\left(U^{\prime} V^{\prime} W^{\prime}-0\right)$,

3) le iperquadriche rolonde intorno ad un loro piano principale (per es. $W^{\prime}=0$ ),

4) le iperquadriche rotonde intorno a due piani principali (sghembi) (per es. $U^{\prime}=V^{\prime}=0$ ),

5) le ipersuperficic a curvotura nulla (applicabili su $S_{3}$;

$$
U^{\prime}=V^{\prime}=W^{\prime \prime}=0 \text { ). }
$$

12. Possiamo giovarei dei calcoli eseguiti, modificandoli lievemente, per la determinazione delle ipersuperficie esistenti in uno spazio a 4 dimensioni a curratura costante $K$, e aventi il solito $d s^{2}$.

Si ricordi a tal fine che le equazioni di Gauss vengono modificate solo in guanto nei secondi membri al posto dei simboli di Rumann $(x \beta, \gamma \delta)$ bisogna leggere $(\alpha \beta, \gamma \delta)-\dot{K}\left(\alpha_{\alpha \gamma} a_{\beta \delta}-a_{\alpha \delta} a_{\beta \gamma}\right)$; quindi le $\left(G_{2}\right)$ restano inalterate, mentre nelle $\left(G_{1}\right)$ al posto di $(i k, i k)$ va messo $\left(i h_{i}, i k\right)-K H_{i} H_{k}$. Le equazioni di Conazzr restano formalmente inalterate pur di intendere che in esse, p. es. nella forma scritta al n. 6, si abbia

$$
A_{i}=(j l, j l)-K H_{j} H_{l} \quad(i-:=j-\mid=l) .
$$

Fra queste $A_{1}$ vale ancora la relazione $A_{1}+A_{2}=A_{3}$, quindi 0 sono tutt'e tre nulle, e allora la $V_{3}$ è a curvatura relativa (all'ambiente) nulla, oppure sono tutte diverse da zero.

Il processo d'integrazione delle equazioni di Codazzr resta inalterato e conduce per le $A_{i}$ alle stesse espressioni ottenute nei un. 7,8 .

Nel caso del n. 7, col significato attuale delle $A$, si ha

$(12,12)=\left(K^{r}+h\right) H_{1} H_{2} ; \quad(23,23)=(K+h) I_{2} H_{3} ; \quad(31,31)=(K+h) H_{3} H_{2}$ quindi si tratta di $V_{3}$ a curvatura costante (assoluta $K: h$, relativa $h$ ). 
Infine nel caso del 11. 8 (sempre per $U^{\prime} V^{\prime} W^{\prime} 0$ ) bisogna integrare il sistema di equazioni che nasce dal sostituire ai primi membri delle equazioni del n. $\bar{y}$ le espressioni dei simboli di Rimmañ tratte dalle $A_{i}$, cioè p. es.:

$$
(23,23)=\frac{H \cdot U}{(U V W)^{2}} I_{2} H_{3}+\kappa H_{2} H_{3}
$$

II sistema così ottenuto è completamente integrabile; una delle conseguenze algebrico-differenziali è

$$
\begin{aligned}
& -1 \frac{(W-V)^{2}}{H_{1}} U^{\prime \prime}+\frac{1}{2} V^{\prime \prime}-\frac{1}{2} W^{\prime \prime}=
\end{aligned}
$$

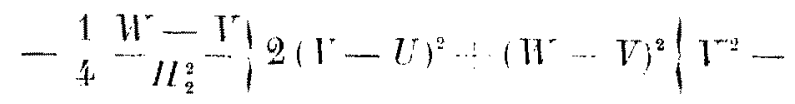

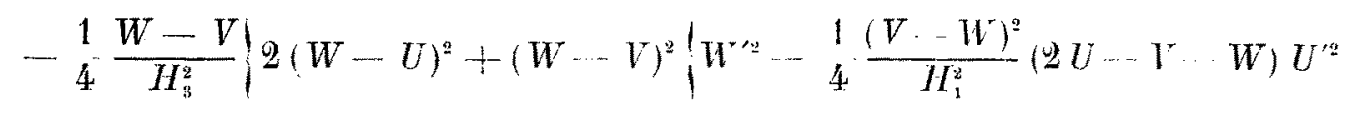

$$
\begin{aligned}
& +\frac{h}{(U V W)^{2}}(W-V)^{2}\left(U^{2}-V W\right)+K(W-V)^{2}(2 U-V \cdots W)
\end{aligned}
$$

e questa successivamente derivata rispetto ad $u$ dà

$$
\left[\begin{array}{c}
1 \\
U^{\prime}
\end{array}\left(\frac{U^{\prime \prime \prime}}{U^{\prime}}\right)\right]^{\prime}=-48 h \frac{U^{\prime}}{U^{\prime \prime}}-48 K U^{\prime}
$$

da cui integrando

$$
\int \frac{\sqrt{U} d U}{\sqrt{-4 K U^{b}+\alpha_{1} U^{4}+\alpha_{2} U^{3}+\alpha_{3} U^{2}+\alpha_{4} U-4 h}}=u
$$

e similmente per $V$ e $W$.

Trovato l'elemento lineare si può anche in questo caso esaurire completamente la ricerca e rendere più espressivo il risultato costruendo eftettivamente il modello (unico, per la sua rigidità) di $V_{3}$ che lo possiede.

Supponiamo l'ambiente a curvatura positiva unitária, $K=1$, in modo da potercelo raffigurare nell'ipersfera $V_{4}^{2}$ di equazione

$$
\sum_{i}^{5} x_{i}^{2}=1
$$

di uno $S_{s}$ enclideo. 
Dico che le nostre $V_{3}$ sono segate su di essa dagli iperconi che hanno il vertice nel centro dell'ipersfera (sono dunque le analoghe delle coniche sferiche). Basta costruirsi il $d s^{2}$ per queste $V_{s}$ e confrontarlo con quello ultimamente trovato.

Insieme all'ipersfera

consideriano i coni

$$
\sum_{i} x_{i}^{2}=1
$$

$$
\stackrel{5}{i}_{i} \frac{x_{i}^{2}}{a_{i}-\lambda}=0
$$

Dall'identità $\left({ }^{i}\right)$

$\bigsqcup_{i} x_{i}^{2}\left(\alpha_{i+1}-\lambda\right)\left(\alpha_{i+2}-\lambda\right)\left(\alpha_{i+3}-\lambda\right)\left(\alpha_{i+4}-\lambda\right)=\left(\lambda-\lambda_{1}\right)\left(\lambda-\lambda_{2}\right)\left(\lambda-\lambda_{3}\right)\left(\lambda-\lambda_{4}\right)$

si trae (come nel solito calcolo per la determinazione delle coordinate ellittiche)

$$
x_{i}^{*}=\frac{\left(a_{i}-\lambda_{1}\right)\left(\alpha_{i}-\lambda_{2}\right)\left(a_{i}-\lambda_{3}\right)\left(a_{i}-\lambda_{4}\right)}{P_{i}}
$$

con

$$
P_{i}=\left(a_{i}-a_{i+1}\right)\left(a_{i}-a_{i+2}\right)\left(a_{i}-a_{i+8}\right)\left(a_{i}-a_{i+1}\right)
$$

quindi sopra una delle $V_{3}$ in esame, ciò̀ sopra l'intersezione dell'ipersfera con un rleterminato cono, per es. $\lambda_{4}=0$,

$$
x_{i}^{2}=\frac{\alpha_{i}}{P_{i}}\left(\left(\alpha_{i}-\lambda_{1}\right)\left(\alpha_{i}-\lambda_{2}\right)\left(\alpha_{i}-\lambda_{3}\right) ;\right.
$$

$\lambda_{1}, \lambda_{2}, \lambda_{3}$ saranno le coordinate curvilinee dei punti della $V_{3}$.

Ma dall'identità precedente, per $\lambda_{4}=0$, si ha su tutta la $V_{3}$

$$
\sum x_{i}^{2} a_{i+\mathrm{i}} a_{i+2} a_{i+3} a_{i+4}=0
$$

ciò̀

$$
\sum \frac{1}{P_{i}}\left(a_{i}-\lambda_{1}\right)\left(a_{i}-\lambda_{2}\right)\left(a_{i}-\lambda_{3}\right)=0
$$

quindi

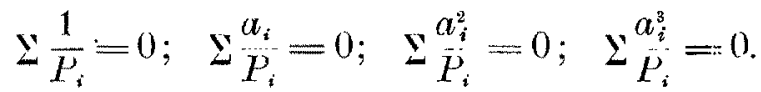

Ancora dalla stessa identita, uguagliando i coefficienti di $\lambda$ nei due

(i) S'intende. che se $i+j>5, \alpha_{i j}=a_{i+j-j}$. 
membri, si ha

$$
\sum x_{i}^{2}\left(a_{i+1} a_{i+2} a_{i+3}+a_{i+1} a_{i+2} a_{i+4}+a_{i+3} a_{i+3} a_{i+4}+a_{i+2} a_{i+3} a_{i+4}\right)=\lambda_{1} \lambda_{2} \lambda_{3}
$$

dalla quale segue fra l'altro

$$
\sum \frac{a_{i}^{4}}{P_{i}}=1
$$

Le identità rilevate per le $a_{i}$ permettono di calcolare facilmente il $d s^{2}$. Per esempio il coefficiente di $d \lambda_{1}^{2}$ vale

$$
\frac{1}{4} \frac{\sum a_{i}\left(a_{i}-\lambda_{2}\right)\left(a_{i}-\lambda_{3}\right)\left(a_{i+1}-\lambda_{1}\right)\left(a_{i+2}-\lambda_{1}\right)\left(a_{i+3}-\lambda_{1}\right)\left(a_{i+4}-\lambda_{1}\right)}{\left(a_{1}-\lambda_{1}\right)\left(a_{2}-\lambda_{1}\right)\left(a_{3}-\lambda_{1}\right)\left(a_{4}-\lambda_{1}\right)\left(a_{5}-\lambda_{1}\right)}
$$

cioè per le precedenti

$$
\frac{\left(\lambda_{1}-\lambda_{2}\right)\left(\lambda_{1}-\lambda_{3}\right) \dot{\lambda}_{1}}{4 Q_{2}\left(\lambda_{1}\right)}
$$

$Q_{5}$ essendo un polinomio di quinto grado in $\lambda_{1}$; analogamente si hanno $i$ coefficienti di $d \lambda_{2}^{2}, d \lambda_{3}^{2}$; l'elemento lineare così ottenuto è proprio del tipo richiesto. Dunque:

Le nostre $V_{3}$ negli $S_{4}$ a curvatura costante positiva $K$ sono segate sull'ipersfera di $S_{\hbar}$, di raggio $\sqrt{K}$, dai coni quadrici col vertice nel centro di essa.

Sono insomma le $V_{3}$ che per proiezione stereografica dell'ipersfera sopra un $S_{4}$ euclideo danno luogo alle $V_{3}$ analoghe alle ciclidi.

Rimarrebbero da esaminare $i$ casi $U^{\prime} V^{\prime} . !=0, W^{\prime}=0$, e $U^{\prime}=V^{\prime}=0$, $W^{\prime}=0$; i risultati dei $u n{ }^{i} 9,10$ vengono modificati solo in quanto i polinomi che figurano a denominatore degli integrali (iperellittici nel primo caso, ellittici nel secondo) aumentano il loro grado di un'unità (quindi sono di grado 4 e 3 rispettivamente). Analoga alla precedente è la costruzione dei modelli aventi questi elementi lineari. 
$\S$ 4. $V_{3}$ mistenti in $S_{4}$ euclideo o a curvatuha costante CON ELEMENTO LINEARE

$$
d s^{*}=\Lambda(u, v)\left(d u^{2}+d v^{2}\right)(k-W)+d w^{2} .
$$

13. Dalle espressioni dei simboli di Riemann per il $d s^{2}$ precedente si hanno le equazioni di Gauss:

$$
\begin{aligned}
& \Omega_{11} \Omega_{22}-\Omega_{12}^{2}=-\frac{1}{2} \Lambda \cdot(k-W)\left(\frac{\partial^{2} \log \Lambda}{\partial u^{2}}+\frac{\partial^{2} \log \Lambda}{\partial v^{2}}\right)- \\
& -\frac{1}{4} \Lambda^{2} W^{\prime 2}=(12,12) \\
& \Omega_{22} \Omega_{33}-\Omega_{23}^{2}=\Omega_{93} \Omega_{13}-\Omega_{31}^{2}=-\frac{1}{4} \frac{\Lambda W^{\prime 3}}{k-W}+ \\
& +\frac{1}{2} \Lambda W^{\prime \prime}=(23,23)=(31,31) \\
& \Omega_{13} \Omega_{23}-\Omega_{13} \Omega_{23}=\Omega_{23} \Omega_{81}-\Omega_{21} \Omega_{83}=\Omega_{31} \Omega_{12}-\Omega_{32} \Omega_{11}=0 .
\end{aligned}
$$

Qui, esclusa la soluzione evidente delle $V_{3}$ applicabili su $S_{3}$, sono possibili due casi: o $(12,12)(23,23)(31,31)=\mid=0$, oppure $(12,12)=\mid=0(23,23)=$ $=(31,31)=0$.

14. Mettiamoci nella prima ipotesi. Allora (n. 3, I) sono

$$
\Omega_{12}=\Omega_{23}=\Omega_{31}=0 ; \quad \Omega_{11}^{2}=\Omega_{22}^{2}=(12,12) ; \quad \Omega_{33}^{2}=\frac{(13,13)^{2}}{(12,12)} .
$$

Le equazioni di Codazzi divengono

$$
\begin{aligned}
& \frac{\partial \log \Omega_{11}}{\partial v}=\frac{\partial \log \Lambda}{\partial v} ; \quad \frac{\partial \log \Omega_{11}}{\partial w}=-\frac{1}{2} \frac{W^{\prime}}{k-W}\left|1+\Lambda(k-W) \frac{\Omega_{s 3}}{\Omega_{11}}\right| \\
& \frac{\partial \log \Omega_{11}}{\partial u}=\frac{\partial \log \Lambda}{\partial u} ; \quad \frac{\partial \log \Omega_{11}}{\partial w}=-\frac{1}{2} \frac{W^{\prime}}{k-W}\left|1+\Lambda(k-W) \frac{\Omega_{s 3}}{\Omega_{11}}\right| \\
& \frac{\partial \Omega_{33}}{\partial u}=\frac{\partial \Omega_{33}}{\partial v}=0
\end{aligned}
$$


e danno $\Omega_{33}=\Omega_{33}(w) ; \Omega_{11}=\Lambda \cdot p(w)$, e per $\varphi(w)$ l'equazione differenziale

$$
\frac{d \log \varphi(w)}{d w}=-\frac{1}{2} \frac{W^{\prime}}{k-W}\left|1+(k-W) \frac{\Omega_{3 z}}{\varphi(w)}\right|
$$

Bisogna vedere se si può soddisfare a queste con le espressioni delle $\Omega_{i i}$ fornite dalle equazioni di Gauss. Poichè $\Omega_{33}$ è funzione della sola $w$, tale dev'essere pure

$$
\frac{1}{\Omega_{33}^{2}}=\frac{(12,12)}{(13,13)^{2}}=\frac{-\frac{k-W}{2 \Lambda}\left(\frac{\partial^{2} \log \Lambda}{\partial u^{2}}+\frac{\partial^{2} \log \Lambda}{\partial v^{2}}\right)-\frac{W^{\prime 2}}{4}}{\left(\frac{1}{4} \frac{W^{\prime 2}}{k-\bar{W}}+\frac{W^{\prime \prime}}{2}\right)^{2}}
$$

e perciò occorre e basta che sia $\Lambda$ soluzione dell'equazione

$$
-\frac{1}{2 \Lambda}\left(\frac{\partial^{2} \log \Lambda}{\partial u^{2}}+\frac{\partial^{2} \log \Lambda}{\partial v^{2}}\right)=c(\text { costante })
$$

L'interpretazione geometrica è immediata; l'espressione a destra divisa per $k-W$ dà la curvatura di una superficie $w=$ cost. nel punto $(u, v)$ : essa è dunque costante $\left(=\frac{c}{k-W}\right)$.

Si ha quindi

$$
\Omega_{83}=\frac{\frac{1}{4} \frac{W^{\prime 2}}{k-W}+W^{\prime \prime}}{\sqrt{c(k-W)-\frac{1}{4} W^{\prime \prime}}}
$$

e perciò dev'essere $c>0$, anzi tale da rendere positivo il radicando.

Dall'equazione di Gavss rimasta si ha finalmente

$$
\Omega_{11}=\Omega_{32}=\Lambda \sqrt{c(k-W)-\frac{1}{4} W^{\prime 2}} ; \quad \varphi(w)=\sqrt{c(k-W)-\frac{1}{4} W^{\prime 2}} .
$$

Se si portano queste espressioni nell'equazione differenziale in $\varphi(w)$ si trova ch'essa è identicamente soddisfatta, quindi $W$ non è soggetta ad alcuna condizione.

Si può domandare se fra queste $V_{3}$ (indeformabili in $S_{4}$ ) ve ne siano 
a curvatura costante $h$ (necessariamente $h>0$ ). Deve essere in tal caso:

$$
\begin{gathered}
\left.(12,12)=\Lambda^{2} \mid c(k-W) \cdots \frac{1}{4} W^{\prime 2}\right\}=h \Lambda^{3}(k-W)^{2} \\
(31,31)=(23,23)=\Lambda\left\{\frac{1}{4} k \frac{W^{\prime 2}}{k-W}+\frac{1}{2} W^{\prime \prime}\right\}=h \Lambda(k-W)
\end{gathered}
$$

si hanno quindi le due equazioni differenziali in $W$ :

$$
\begin{aligned}
& \frac{1}{4} W^{\prime 2}+h(k-W)^{2}-c(k-W)=0 \\
& \frac{1}{2} W^{\prime \prime}+\frac{1}{4} \frac{W^{\prime 2}}{k-W}-h(k-W)=0
\end{aligned}
$$

Ma la prima è un integrale primo della seconda; infatti derivandola e separando il fattore $W^{\prime}=1=0$, si ha

$$
\frac{1}{2} W^{\prime \prime}-2 h(k-W)=-c
$$

e sostituendo nella prima stessa e dividendo per $k-W_{-}=0$ si ottiene appunto la seconda. Eseguendo l'integrazione della prima, e prescindendo da costanti inessenziali si ha

$$
k-W=\frac{c}{k} \operatorname{sen}^{2} \sqrt{h} w
$$

(si potrebbe far a meno di $h$ passando ad un $d s^{2}$ simile).

15. Gli elementi lineari del $n .^{0}$ precedente appartengono a $V_{3}$ di rotazione intorno ad un asse (l'ultimo, in particolare, ad una ipersfera); il profilo meridiano, data l'arbitrarietà di $W$, è arbitrario.

Per la forma (2') dell'elemento lineare trasformato di (2) si può anche per esso costruire una $V_{3}$ di rotazione in $S_{4}$ che lo possegga.

16. Supponiamo $(23,23)=0,(31,31)=0$.

Si ha

$$
\frac{1}{2} \frac{W^{\prime 2}}{k-W}+W^{\prime \prime}=0
$$


da cui integrando, per $W^{\prime}+$, e prescindendo da costanti inessenziali

$$
k-W=w^{2} .
$$

Inoltre per l'annullarsi di quei due simboli deve essere

$$
\Omega_{13}=\Omega_{23}=\Omega_{33}=0 ; \quad \Omega_{11} \Omega_{29}-\Omega_{12}^{2}=(12,12) .
$$

Le equazioni di Codazzı non identicamente soddisfatte sono

$$
\begin{aligned}
& \frac{\partial \Omega_{11}}{\partial v}-\frac{\partial \Omega_{12}}{\partial u}=\frac{1}{2} \frac{\partial \log \Lambda}{\partial v}\left(\Omega_{11}+\Omega_{22}\right) ; \quad \frac{\partial \Omega_{11}}{\partial w}=\frac{\Omega_{11}}{w} \\
& \frac{\partial \Omega_{32}}{\partial u}-\frac{\partial \Omega_{12}}{\partial v}=\frac{1}{2} \frac{\partial \log \Lambda}{\partial u}\left(\Omega_{22}+\Omega_{11}\right) ; \frac{\partial \Omega_{22}}{\partial w}=\frac{\Omega_{22}}{w} .
\end{aligned}
$$

Si ha intanto

$$
\Omega_{11}=\varphi_{1}(u, v) w ; \quad \Omega_{22}=\varphi_{2}(u, v) u
$$

e perciò dall'ultima equazione di Gauss

$$
\Omega_{12}=w \sqrt{\varphi_{1} \varphi_{2}+\Lambda^{3}+\frac{\Lambda}{2}\left(\frac{\partial^{2} \log \Lambda}{\partial u^{2}}+\frac{\partial^{2} \log \Lambda}{\partial v^{2}}\right)}=w \sqrt{\Delta}
$$

e per le equazioni di Cosazzi rimaste

$$
\begin{aligned}
& \partial \varphi_{1}=\frac{\partial \sqrt{\Delta}}{\partial u}+\frac{1}{2} \frac{\partial \log \Lambda}{\partial v}\left(\varphi_{1}+\varphi_{2}\right) \\
& \partial v=\frac{\partial \sqrt{\Delta}}{\partial v}+\frac{\partial \log \Lambda}{\partial u}\left(\varphi_{1}+\varphi_{2}\right) .
\end{aligned}
$$

Può forse convenire di assumere come incognite $\theta_{1}=\stackrel{\varphi}{\Lambda}_{\Lambda}$ e $\theta_{2}=\frac{\dot{\varphi}_{9}}{1} ;$ indicando ora con $k$ la curvatura di $\boldsymbol{\Lambda}\left(d u^{2}+d v^{2}\right)$ si ha

$$
\begin{aligned}
& \frac{\partial \theta_{1}}{\partial v}=\frac{1}{\Lambda} \frac{\partial \Lambda \sqrt{\theta_{1} \theta_{2}+1-k}}{\partial u}-\frac{1}{2}\left(\theta_{1}-\theta_{2}\right) \frac{\partial \log \Lambda}{\partial v} \\
& \frac{\partial \theta_{2}}{\partial u}=\frac{1}{\Lambda} \frac{\partial \Lambda \sqrt{\theta_{1} \theta_{2}+1-k}}{\partial v}+\frac{1}{2}\left(\theta_{1}-\theta_{2}\right) \frac{\partial \log \Lambda}{\partial u} .
\end{aligned}
$$

Ad ogni soluzione $\left(\theta_{1}, \theta_{2}\right)$ di questo sistema corrisponde una $V_{3}$ del nostro tipo, certo deformabile perchè le espressioni delle $\Omega_{r s}$, soddisfacenti 
alle equazioni di Gauss-Codazi, non sono determinate in modo unico dalle equazioni stesse.

Del resto l'effettiva esistenza e deformabilità in $S_{4}$ delle $V_{3}$ in esame si prova subito geometricamente.

Sopra un'ipersfera di raggio unitario si costruisca una superficie (iniziale) il cui quadrato dell'elemento lineare sia $d \sigma^{2}=\Lambda(u, v)\left(d u^{2}+d v^{2}\right)$. Il cono $V_{3}$ che la proietta dal centro dell'ipersfera ha proprio

$$
d s^{2}=w^{2} d \sigma^{2}+d w^{3}=\Lambda(u, v)\left(d u^{2}+d v^{2}\right) w^{2}+d w^{2}
$$

quando si assuma come parametro $w$ la distanza del punto che si considera dal centro. Ad ogni flessione della superficie iniziale corrisponde una deformazione per applicabilità della $V_{3}$.

Giò vale qualunque sia $\Lambda$; se però la superficie iniziale ha curvatura gaussiana (assoluta) $=1$, cioè se è a curvatura (relativa) nulla considerata entro l'ipersfera (e solo in questo caso) la varietà risulta applicabile su $S_{3}$. Basta provare che se e solo se

$$
\frac{\partial^{2} \log \Lambda}{\partial u^{2}}+\frac{\partial^{2} \log \Lambda}{\partial v^{2}}=-2 \Lambda
$$

è $(12,12)=0$; il che è immediato essendo ora $k-W=w^{2}$.

Ci si può domandare se insieme ad una $V_{3}$ del tipo esaminato esista in $S_{4}$ anche una sua trasformata geodetica.

Poichè al

$$
d s^{2}=\Lambda(u, v)\left(d u^{2}+d v^{2}\right)(k-W)+d w^{2}
$$

corrisponde l'altro

$$
d s^{\prime 2}=\Lambda(u, v)\left(d u^{2}+d v^{2}\right)_{k W}^{k-\cdots W}+\frac{d w^{2}}{W^{2}}
$$

per le nostre $V_{s}\left(W=k-t^{2}\right)$ si ha

$$
\text { a. } s^{\prime \prime}=\Lambda(u, v)\left(d u^{2}+a v^{2}\right) \frac{w^{2}}{k\left(k-w^{2}\right)}+\frac{d w^{2}}{\left(k-w^{2}\right)^{2}} \quad(k \gtrless 0) .
$$

Per vedere se questo $d s^{\prime}$ può esistere in $S_{*}$ è opportuno cambiare il parametro $w$ in modo da poter confrontare il $d s^{\prime}$ trasformato con quelli già riconosciuti esistenti in $S_{1}$. 
Posto $d t=\frac{d w}{a^{2}-w^{2}}$ se $k=a^{2}>0$, ovvero $d t=\frac{d w}{a^{2}+w^{2}}$ se $k=-a^{2}$ si ha

$$
d s^{\prime 2}=\Lambda(u, v)\left(d u^{2}+d v^{2}\right) \frac{\operatorname{senh}^{2} a t}{a^{2}}+d t^{2}
$$

oppure

$$
d s^{2}=\Lambda(u, v)\left(d u^{2}+d v^{2}\right) \frac{\operatorname{sen}^{2} a t}{a^{2}}+d t^{2}
$$

Nessuno di. questi elementi lineari rientra nei tipi trovati, almeno in generale; nou nell'ultimo per la presenza del $\operatorname{senh}^{2}$ at o del $\operatorname{sen}^{2} a t$; non nei precedenti essendo ora $\Lambda$ qualsiasi.

Però se $\Lambda$ è tale da dare a $\Lambda\left(d w^{3}+d v^{2}\right)$ curvatura costante, il secondo $d s^{\prime}$ appartiene, come s'è trovato, ad un'ipersfera.

17. Rimane da esaminare l'ipotesi $W=$ costante. ì evidente che in questo caso si ha un modello del $d s^{2}$ in un cilindro di $S_{4}$ la cui sezione retta (in $S_{s}$ ) ha l'elemento $d s^{2}=c_{\Lambda}(u, v)\left(d u^{2}+d v^{2}\right), c>0$.

Queste $V_{s}$ ammettono non soltanto trasformazioni geodetiche, ma anche per parallelismo di Levrarvita $\left.{ }^{8}\right)$. Se in particolare il $d s^{2}$ è a curvatura mulla le $V_{3}$ corrispondenti sono applicabili su $S_{3}$.

18. Riassumendo:

Le $V_{3}$ di elemento lineare $d s^{2}=\Lambda(u, v)\left(d u^{2}+d v^{2}\right)(k-W)+d w^{2}$ esistenti in $S_{4}$ euclideo sono:

1) ipersuperficie rotonde intorno ad una retta (rigide; $W$ arbitrario).

2) ipersuperficie coniche o cilindriche (deformabili; I arbitrario); queste ammettono trasformate geodetiche in $S_{4}$ solo quando le superficie $w=$ cost. sono a curvatura costante.

3) applicabili su $S_{3}$.

19. Se l'ambiente ha curvatura costante $K_{0}=0$ si hanno risultati del tutto simili. Sempre i secondi membri delle $\left(G_{2}\right)$ sono nulli, mentre nelle $\left(G_{1}\right)$ al posto di $(12,12)$ e di $(23,23)=(13,13)$ bisogna leggere rispettivamente $(12,12)-K_{0} \Lambda^{2}(k-W)^{2}$ e $(23,23)-K_{0} \Lambda(k-W)$. Si presentano quindi gli stessi casi precedenti.

(8) Cfr. la mia Nota: Le trasformazioni puntuali fra varietà che conservano il parallelismo di Levi-Civita [Rend, Accad. Lincel, 19201]. 
Quando tutte le $\Omega_{i k}$ sono determinate dal $d s^{2}$ si ha

$$
\begin{gathered}
\Omega_{12}=\Omega_{23}=\Omega_{31}=0 \\
\Omega_{14}=\Omega_{23}=\Lambda(u, v) \sqrt{c(k-W)-K_{0}(k-W)^{2}-\frac{1}{4} W^{\prime 2}} \\
\Omega_{33}=\frac{1 W^{\prime 2}}{\sqrt{c(k-W)-W_{0}}+W^{\prime \prime}-K_{0}\left(k-W^{2}\right)^{2}}
\end{gathered}
$$

essendo $c$ la curvatura costante del $d s^{2}=\Lambda\left(d u^{2}+d v^{2}\right)$ e $W$ affatto arbitraria.

Quando ciò non accade $\left(\Omega_{28} \Omega_{39}-\Omega_{23}^{2}=\Omega_{11} \Omega_{39}-\Omega_{13}^{2}=0\right)$ rimane $\Lambda(u, v)$ arbitraria, mentre si ha $k-W=\cos ^{2}\left(\sqrt{K_{0}} w\right)$ oppure $=\cosh ^{2}\left(\sqrt{-\bar{K}_{0}} w\right)$ secondo che $K_{0}>0$ oppure $K_{0}<0$. 